\title{
GLUEBALL MASS ESTIMATE FROM FINITE TEMPERATURE SU(2) LATTICE STUDIES
}

\author{
J. ENGELS, F. KARSCH and H. SATZ \\ Department of Theoretical Physics, University of Bielefeld, Germany
}

and

\author{
I. MONTVAY \\ II. Institut für Theoretische Physik ${ }^{1}$, Universität Hamburg, Germany
}

Received 3 April 1981

Finite temperature Monte Carlo simulations of the SU(2) Yang-Mills system on the lattice are used to obtain an estimate of the mass $m_{\mathrm{G}}$ of the lowest gluonium state. Taking gluon matter in the hadronic regime, below the deconfinement transition, to follow the usual string or bag model pattern, we find from the temperature dependence of the energy density and of the specific heat that $m_{\mathrm{G}}=(1.7 \pm 0.5) \sqrt{\sigma}$, where $\sigma$ is the string tension.

Monte Carlo studies of the SU(2) Yang-Mills system on the lattice at finite physical temperature $T$ have revealed a deconfinement transition [1-3] at the critical temperature $T_{\mathrm{c}} \approx 0.5 \sqrt{\sigma}$, where the system changes from gluonium matter to gluon gas; here $\sigma$ denotes the strong tension. Above $T_{\mathrm{c}}$, at sufficiently high temperatures, this gluon gas attains the (parameter-free) Stefan-Boltzmann limit of an ideal massless boson gas [3]. Below $T_{\mathrm{c}}$, the behaviour of the system is less well understood; but if we believe that it also provides there a reasonable approximation to the real quark-gluon world, then we expect gluonium matter to exhibit the same basic features as hadron matter.

The description of hadrons as bound states of quarks leads to a resonance spectrum, starting with a lowest state of mass $m_{0}$. In the continuum, both bag [4] and dual string [5] models predict the density $\tau(m)$ of reso. nance states to increase exponentially with increasing resonance mass $m$ and thus yield the form ${ }^{\neq 1}$

$$
\begin{aligned}
& \tau(m)=d \delta\left(m-m_{0}\right)+c \theta\left(m-2 m_{0}\right) m^{-a} \mathrm{e}^{b m}, \\
& a, b, c, d=\mathrm{const} .
\end{aligned}
$$

1 Supported by the Bundesministerium für Forschung und Technologie, Bonn.

$\neq 1$ We have ignored here the possibility of discrete excited states below the continuum threshold $m=2 m_{0}$. first obtained in the statistical bootstrap model [6] . While the constant $b$ is determined by the bag size or the string tension (Regge slope), $a$ depends more on the details of the model [7]. The normalization $c$ fixes the relative strength of resonance excitation to the $d$ fold degenerate ground state.

It is well known that a density of states of the form (1) is associated with critical behavior [8] : the partition function

$$
\begin{aligned}
& Z(T, V)=\exp \left(\frac{V}{(2 \pi)^{3}} \int_{m_{0}}^{\infty} \mathrm{d} m \tau(m)\right. \\
& \left.\quad \times \int \mathrm{d}^{3} p \exp \left[-T^{-1}\left(p^{2}+m^{2}\right)^{1 / 2}\right]\right),
\end{aligned}
$$

for an ideal gas of resonances, or its derivatives, become singular at the temperature $T_{\mathrm{c}}=1 / \mathrm{b}$; above $T_{\mathrm{c}}$, the integral (2) is no longer defined. If we identify this critical behaviour with the deconfinement transition of the SU(2) lattice problem at $T_{\mathrm{c}}=0.5 \sqrt{\sigma}$, then the approach to deconfinement is governed by the lowest gluonium mass $m_{0}=m_{G}$ as scale. Comparing the form predicted by eq. (1) with that obtained from the Monte Carlo simulation of the SU(2) Yang-Mills system thus gives an estimate of the glueball mass $m_{\mathrm{G}}$.

We note that this approach - using the resonance 
spectrum of hadron physics to determine the glueball mass - provides an alternative to correlation length estimates of $m_{\mathrm{G}}[9-11]$. In the field-theoretic confinement problem, i.e. for the $T=0$ case using a symmetric space-time lattice, the correlation function $\rho(r)$ for two lattice plaquettes separated by $r$ links is expected to vanish exponentially for large $r$,

$\rho(r) \sim \exp -(r / \xi)$.

In the scaling limit of vanishing bare coupling, $g^{2} \rightarrow 0$, the dimensionless correlation length $\xi$ must diverge and the lattice spacing $a$ go to zero such that

$m_{\mathrm{G}} \equiv 1 / \xi a$,

approaches a finite limit, interpreted as lowest "boundstate" mass of the theory. Hence Monte Carlo calculations of $\rho(r)$ for sufficiently large $r$ over a range of sufficiently small $g^{2}$, when compared with the form (3), yield an estimate for $m_{\mathrm{G}}$. An evaluation of SU(2) results gave [9] $m_{\mathrm{G}}=(3.7 \pm 1.2) \sqrt{\sigma}$; the relation $\sigma$ $=1 / 2 \pi \alpha^{\prime}$ between $\sigma$ and the Regge slope $\alpha^{\prime}$, together

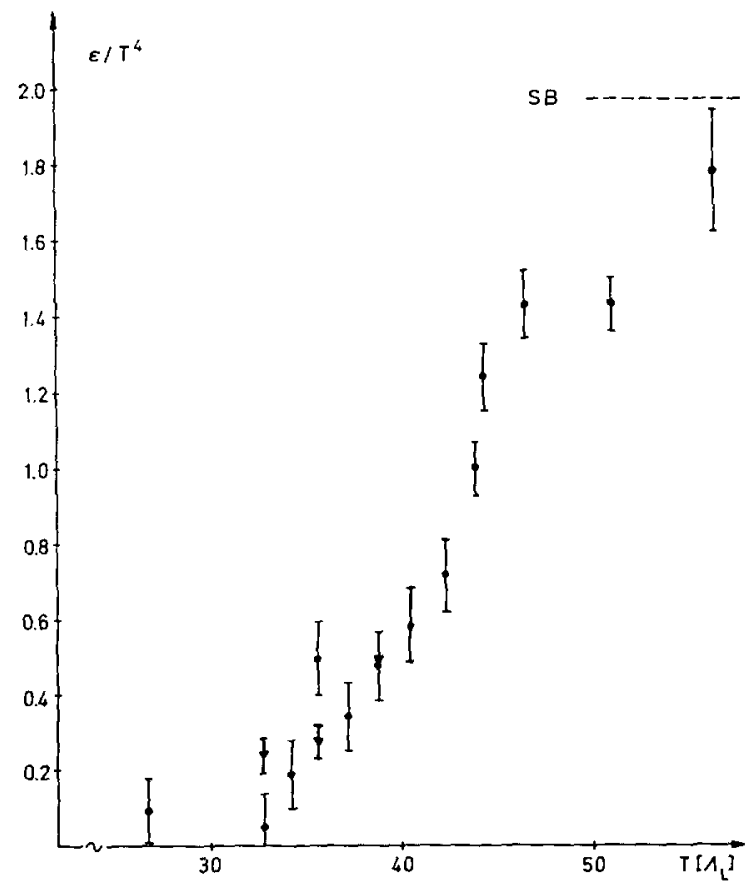

Fig. 1. Energy density versus temperature in the critical region; the dashed line (SB) is the Stefan-Boltzmann limit $\epsilon / T^{4}$ $=\pi^{2} / 5$. The triangular points are from calculations using a $9^{3} \times 3$ lattice.

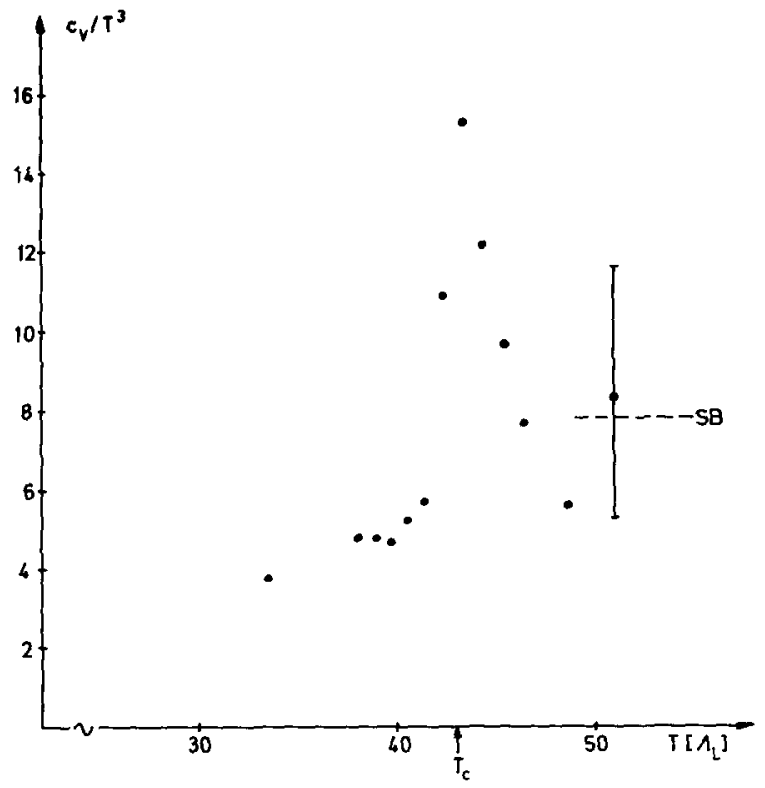

Fig. 2. Specific heat versus temperature in the critical region; the dashed line (SB) is the Stefan-Boltzmann limit $C_{V} / T^{3}$ $=4 \pi^{2} / 5$. On the highest temperature point we show typical error bars.

with the empirical value $\alpha^{\prime}=1 \mathrm{GeV}^{-2}$, then implies $m_{\mathrm{G}}=1.5 \pm 0.5 \mathrm{GeV}$. Results from a simulation using the icosahedral subgroup $[10,12]$ of $S U(2)$ agree with this value. An evaluation based on the analytic continuation of the strong coupling expansion [11] leads to a somewhat lower value, with $m_{\mathrm{G}}=(1.8 \pm 0.8) \sqrt{\sigma}$.

Returning now to finite-temperature thermodynamics, we show in figs. 1 and 2 or SU(2) Monte Carlo results for energy density and specific heat as function of the temperature. To obtain them, we have used a $7^{3} \times 3$ lattice, corrected for finite-size effects [13]. The connection between $g^{2}$ and $a$ is given by the renormalization group; the temperature is $T=1 / 3 a$, since we have three lattice sites in the temperature direction. The lattice spacing is taken to be the same in all directions. The data shown are based on a larger number of iterations (about 3000 per point) than our previous work [3]; the resulting critical temperature is with $T_{\mathrm{c}} \approx 43 \Lambda_{\mathrm{L}}$ somewhat higher than the $40 \Lambda_{\mathrm{L}}$ found there. We note that both energy density and specific heat values lie near the asymptotic StefanBoltzmann limits already at $T \approx 50 \Lambda_{\mathrm{L}}$.

The region of interest in the present problem is that of temperatures just below $T_{c}$, where we expect 
gluonium matter, consisting dominantly of the various excited glueball resonances. As $T \rightarrow 0$, the system will contain more ground states (glueballs) and fewer resonances, eventually becoming an ideal gas of glueballs.

For a gluonium gas characterized by a resonance spectrum of the form (1), we obtain for the energy density

$$
\begin{aligned}
& \epsilon(T)=\epsilon_{\mathrm{G}}(T)+\frac{c T}{2 \pi^{2}} \int_{2 m_{\mathrm{G}}}^{\infty} \mathrm{d} m m^{3-a} \\
& \quad \times \exp \left(m / T_{\mathrm{c}}\right)\left[K_{1}(m / T)+(3 T / m) K_{2}(m / T)\right],
\end{aligned}
$$

with

$$
\begin{gathered}
\epsilon_{\mathrm{G}}(T)=d\left(m_{\mathrm{G}}^{3} T / 2 \pi^{2}\right)\left[K_{1}\left(m_{\mathrm{G}} / T\right)\right. \\
\left.+\left(3 T / m_{\mathrm{G}}\right) K_{2}\left(m_{\mathrm{G}} / T\right)\right]
\end{gathered}
$$

for the energy density of an ideal glueball gas. The specific heat per unit volume is given by

$$
\begin{aligned}
& C_{V}(T)=\frac{1}{T}\left(\epsilon(T)+\frac{1}{2 \pi^{2}} \int_{2 m_{\mathrm{G}}}^{\infty} \mathrm{d} m m^{4} \tau(m)\right. \\
& \quad \times\left[K_{2}(m / T)+(2 T / m) K_{1}(m / T)\right. \\
& \left.\left.\quad+9(T / m)^{2} K_{2}(m / T)\right]\right) .
\end{aligned}
$$

Besides the glueball mass $m_{\mathrm{G}}$, eqs. (5) and (6) still contain the parameters $a, c$ and $d$ of the spectrum (1), $b$ having been identified as $T_{\mathrm{c}}^{-1}$. We shall now see that these are also essentially determined by the form of the Monte Carlo results.

At $T=T_{\mathrm{c}}$, the energy density (5) remains finite only if $a>7 / 2$, as can easily be seen by use of the large-argument approximation of the Hankel functions. Similarly, the specific heat stays bounded for $a>9 / 2$. If we interpret the results of figs. 1 and 2 as an indication of finite $\epsilon$ and a diverging $C_{V}$ at $T=T_{\mathrm{c}}$, then we must have $7 / 2<a \leqslant 9 / 2$. The ground-state degeneracy is chosen as $d=6$, in accord with strong coupling [14] and bag model [15] estimates. Finally, the constant $c$ in eq. (1) is fixed by the value of $\epsilon\left(T_{\mathrm{c}}\right)$. With all other parameters thus determined, we can now com. pare the functional form of $\epsilon(T)$, from eq. (5), and both form and normalization of $C_{V}(\mathrm{~T})$, from eq. (7), with results of our Monte Carlo simulation of the SU(2) Yang-Mills system, in order to estimate $m_{\mathrm{G}}$.

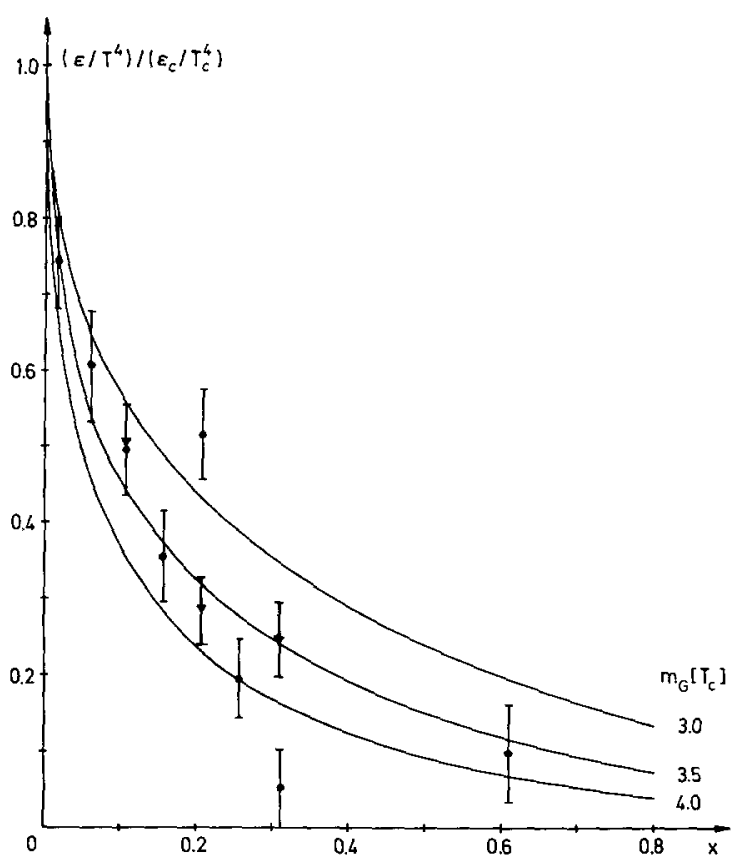

Fig. 3. Energy density, normalized to the critical value $\epsilon_{\mathrm{C}}$ at $T_{\mathrm{c}}$, versus the temperature variable $x=\left(T_{\mathrm{c}} / T-1\right)$; the curves are the resonance gas predictions for $m_{\mathrm{G}}=3.0,3.5$ and $4.0 T_{\mathrm{c}}$. The triangular points are from calculations using a $9^{3} \times 3$ lattice.

In fig. 3, we show the energy density (5) for $a=4$ and $m_{\mathrm{G}} / T_{\mathrm{c}}=3.0,3.5,4.0$, in comparison with the lattice results of $\epsilon$. Both quantities are normalized to the value at $T_{\mathrm{c}}$ and plotted against the dimensionless "temperature" $x=\left(T_{\mathrm{c}}-T\right) / T$. It is seen that $m_{\mathrm{G}}$ $=(3.5 \pm 0.5) T_{\mathrm{c}}$ yields reasonably good agreement. We have repeated this procedure for different values of $a$ in the range $7 / 2<a \leqslant 9 / 2$; to illustrate the results, we show in fig. 4 the forms for $m_{\mathrm{G}}=3.5 T_{\mathrm{c}}$ with $a=3.75$, 4.0 and 4.25 , in comparison with the lattice results. It is evident that a variation of $a$ does not produce very significant changes. Also shown here is the contribution of the pure glueball gas, which is seen to give the dominant contribution for $x \geq 0.5$. Finally we show in fig. 5 the specific heat $C_{V}$ from eq. (7), for the $m_{\mathrm{G}}$ $=3.5$ and 4.0 , with $a=4$. Also here the agreement is found to be reasonable, although at small $x$ the lattice results appear to increase significantly slower than the gluonium gas. This may well be a reflection of the finite lattice size, preventing a true divergence at $x=0$.

Taking into account both the range of $m_{G}$ allowed for a given $a$ and the possible variation with $a$, we con- 


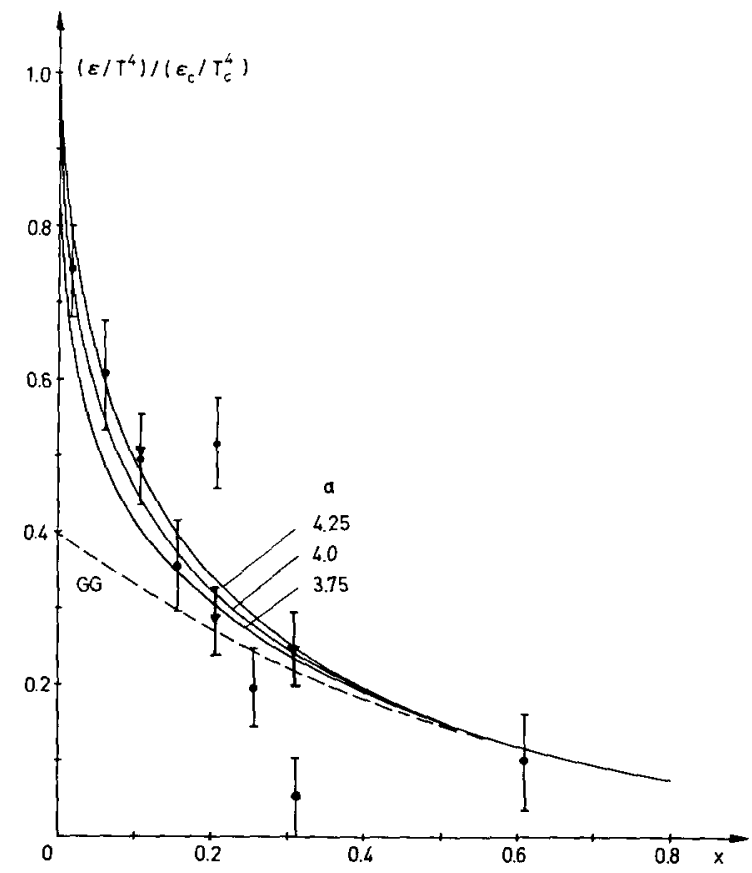

Fig. 4. Energy density, normalized to the critical value $\epsilon_{\mathrm{c}}$ at $T_{\mathrm{c}}$, versus the temperature variable $x=\left(T_{\mathrm{c}} / T-1\right)$; the curves are the resonance gas predictions for $m_{\mathrm{G}}=3.5 T_{\mathrm{c}}$, with $a$ $=3.75,4.0$ and 4.25. The dashed line (GG) is the result of the ideal glueball gas alone. The triangular points are from calculations using a $9^{3} \times 3$ lattice.

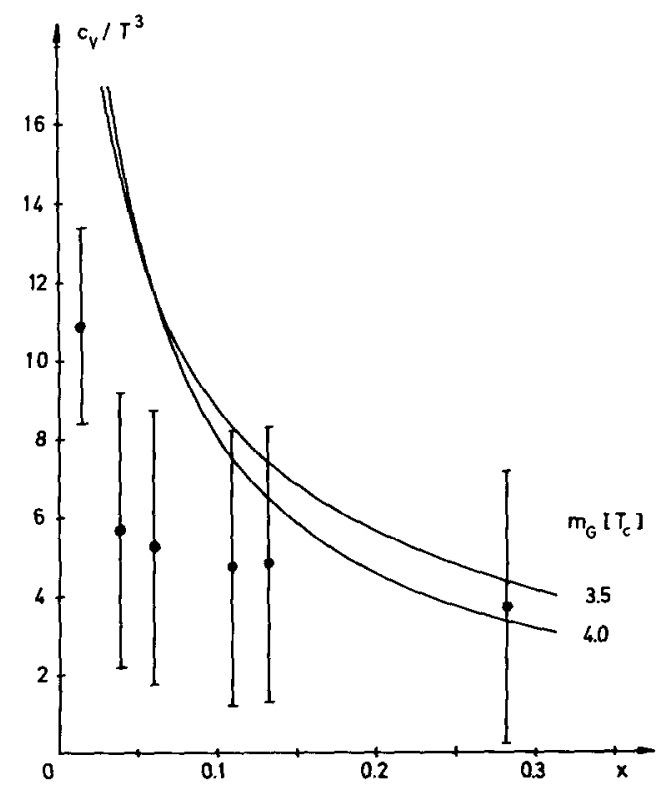

Fig. 5. Specific heat versus $x=\left(T_{\mathrm{c}} / T-1\right)$, with the resonance gas predictions for $m_{\mathrm{G}}=3.5$ and $4 T_{\mathrm{c}}$. The error bars are typical values. clude that $m_{\mathrm{G}}=(3.5 \pm 1.0) T_{\mathfrak{c}}$, which with the relation [10] $\Lambda_{\mathrm{L}}=(0.011 \pm 0.002) \sqrt{\sigma}$ gives us $m_{\mathrm{G}}=(1.7$ $\pm 0.5) \sqrt{\sigma}$. Using the physical value of the string tension $\left(0.16 \mathrm{GeV}^{2}\right)$ in this result based on SU(2) YangMills lattice studies, we would have a glueball mass of $m_{\mathrm{G}}=(0.7 \pm 0.2) \mathrm{GeV}$.

Summarizing, we have presented a method of obtaining an estimate for the mass $m_{G}$ of the lowest gluonium state, based on the expected physical (resonance) features of the confinement regime of gluon mat ter. In closing, we note some further possible sources of error and ways to improve the accuracy of the result. Since the energy density around $T_{\mathrm{c}}$ changes very rapidly, small modifications of the value of $T_{\mathrm{c}}$ can lead to considerable changes in $\epsilon\left(T_{\mathrm{c}}\right)$. Given more accurate lattice calculations, more suitable normalizations appear possible. The degree of "singularity" of $C_{V}$ on the lattice depends of course on the lattice size. Thus simulations using a larger lattice then $7^{3} \times 3$ can provide more reliable points near $T_{\mathrm{c}}$ as well as test the premise that $C_{V}\left(T_{\mathrm{c}}\right)$ diverges. Finally, it would certainly be of interest to see if and how our conclusions are modified in a comparison of eqs. (5) and (7) with the results of SU(2) lattice simulations $[16,17]$

One of us (H.S.) wants to thank L.D. McLerran for a stimulating discussion.

\section{References}

[1] L.D. McLerran and B. Svetitsky, Phys. Lett. 98B (1981) 195.

[2] J. Kuti, J. Polónyi and K. Szlachányi, Phys. Lett. 98B (1981) 199.

[3] J. Engels, F. Karsch, H. Satz and I. Montvay, Phys. Lett. 101 (1981) 89.

[4] A. Chodos, R.L. Jaffe, K. Johnson and V. Weisskopf, Phys. Rev. D9 (1974) 3471.

[5] See e.g.: M. Jacob, ed., Dual theory (North-Holland, Amsterdam, 1974).

[6] See: R. Hagedorn, CERN report 71-12 (Yellow Series) (1971).

[7] K. Huang and S. Weinberg, Phys. Rev. Lett 25 (1970) 855 ;

H. Satz, Phys. Rev. D20 (1979) 582.

[8] N. Cabibbo and G. Parisi, Phys. Lett. 59B (1974) 67.

[9] B. Berg, Phys. Lett. 97B (1980) 401.

[10] G. Bhanot and C. Rebbi, SU (2) string tension, glueball mass and interquark potential by Monte Carlo computations, CERN-TH 2979 (1980).

[11] G. Münster, STrong coupling expansions for the mass gap in lattice gauge theories, Bern preprint BUTP-2/1981 (1981). 
[12] D. Petcher and D.H. Weingarten, Monte Carlo calculations and a model of the phase structure for gauge theories on discrete subgroups of SU (2), Indiana preprint IUHET-53 (1980).

[13] J. Engels, F. Karsch, I. Montvay and H. Satz, Finite lattice effects in Monte Carlo simulations of SU(2) gluon systems, to be published.

[14] J. Kogut, D.K. Sinclair and L. Susskind, Nucl. Phys. B114 (1976) 199.
[15] J. Donoghue, K. Johnson and B. Li, Phys. Lett. 99B (1981) 416.

[16] R.C. Edgar, L. McCrossen and K.J.M. Moriarty, The specific heat of SU (3) lattice gauge theory, Royal Holloway College preprint (1980).

[17] K. Kajantie, C. Montonen and E. Pietarinen, Phase transition of SU(3) gauge theory at finite temperature, Helsinki preprint HU-TFT-81-8 (1981), Z. Phys. C, to be published. 